This document is confidential and is proprietary to the American Chemical Society and its authors. Do not copy or disclose without written permission. If you have received this item in error, notify the sender and delete all copies.

\title{
In situ, heat-induced replacement of GaAs nanowires by Au
}

\begin{tabular}{|r|l|}
\hline Journal: & Nano Letters \\
\hline Manuscript ID & nl-2016-00109z.R1 \\
\hline Manuscript Type: & Communication \\
\hline Date Submitted by the Author: & 08-Apr-2016 \\
\hline Complete List of Authors: & $\begin{array}{l}\text { Fauske, Vidar; Norwegian University of Science and Technology, } \\
\text { Department of Physics } \\
\text { Huh, Junghwan; Norwegian University of Science and Technology, } \\
\text { Department of Electronics and Telecommunications } \\
\text { Divitini, Giorgio; University of Cambridge, Materials Science and Metallurgy } \\
\text { Dheeraj, Dasa; CrayoNano AS } \\
\text { Munshi, A. Mazid; CrayoNano AS, } \\
\text { Ducati, Caterina; University of Cambridge, Materials Science and } \\
\text { Metallurgy } \\
\text { Weman, Helge; Norwegian University of Science and Technology, } \\
\text { Department of Electronics and Telecommunications; CrayoNano AS } \\
\text { Fimland, Bjørn-Ove; Norwegian University of Science and Technology, } \\
\text { Department of Electronics and Telecommunications; CrayoNano AS } \\
\text { van Helvoort, Antonius; Norwegian University of Science and Technology, } \\
\text { Department of Physics }\end{array}$ \\
\hline \hline
\end{tabular}

\section{SCHOLARONE'}

Manuscripts 


\title{
In situ, heat-induced replacement of GaAs nanowires
}

\section{by $\mathrm{Au}$}

\author{
Vidar T. Fauske ${ }^{1}$, Junghwan Huh ${ }^{2}$, Giorgio Divitini ${ }^{3}$, Dasa L. Dheeraj ${ }^{4}$, A. Mazid Munshi ${ }^{4}$, \\ Caterina Ducati ${ }^{3}$, Helge Weman ${ }^{2,4}$, Bjørn-Ove Fimland ${ }^{2,4}$, Antonius T. J. van Helvoort ${ }^{1}$, * \\ ${ }^{1}$ Department of Physics, Norwegian University of Science and Technology (NTNU), NO 7491 \\ Trondheim, Norway \\ ${ }^{2}$ Department of Electronics and Telecommunications, Norwegian University of Science and \\ Technology (NTNU), NO 7491 Trondheim, Norway \\ ${ }^{3}$ Department of Materials Science and Metallurgy, University of Cambridge, Cambridge, CB3 \\ 0FS, United Kingdom \\ ${ }^{4}$ CrayoNano AS, Otto Nielsens vei 12, NO 7052 Trondheim, Norway
}

Here we report on the heat-induced solid state replacement of GaAs by Au in nanowires. Such replacement of semiconductor nanowires by metals is envisioned as a method to achieve well-defined junctions within nanowires. To better understand the mechanisms and dynamics that govern the replacement reaction, we performed in situ heating studies using high-resolution scanning transmission electron microscopy. The dynamic evolution of the phase boundary was investigated, as well as the crystal structure and orientation of the different phases at reaction temperatures. In general, the replacement proceeds one GaAs(111) bi-layer at a time, and no fixed epitaxial relation could be found between the two 


\section{phases. The relative orientation of the phases affects the replacement dynamics and can induce growth twins in the Au nanowire phase. In the case of a limited Au supply, the metal phase can also become liquid.}

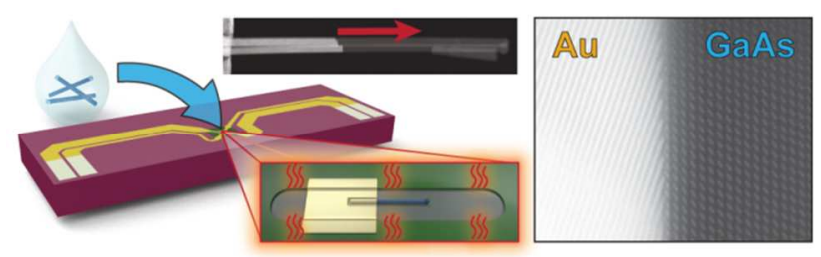

Keywords: Nanowire, solid state replacement, in situ, annealing, GaAs, Au

Semiconductor nanowires are interesting candidates for future electronic and optoelectronic devices $^{1-3}$. In nanowire device applications, metal contacts are typically made to the nanowires, and the performance of the device is often reliant upon the contact properties between the metal and the semiconductor. Normally, a thermal annealing step is performed to improve the contact quality. The interplay between the metal and semiconductor phases at elevated temperatures therefore needs to be understood. Recently, several studies have reported on the heat-induced, solid state replacement of semiconductor nanowires by metal ${ }^{4-11}$. In general, these studies are motivated by improved control of the electrical contact properties. The first report of this phenomena was on the replacement of Si nanowires by metallic nickel silicide (NiSi) ${ }^{4}$. Other material systems studied include $\mathrm{Si}-\mathrm{PtSi}^{5}, \mathrm{Ge}-\mathrm{Cu}_{3} \mathrm{Ge}^{6}, \mathrm{ZnO}-\mathrm{In}^{7}, \mathrm{Ge}-\mathrm{Al}^{8}$, as well as GaAs-Au with varying intermediate metal stacks deposited (Ge/Ni/Ge/Au, $\mathrm{Pd} / \mathrm{Ge} / \mathrm{Au}, \quad \mathrm{Ni} / \mathrm{Ge} / \mathrm{Au}$, $\mathrm{Ni} / \mathrm{Ge} / \mathrm{Au} / \mathrm{Ni} / \mathrm{Au})^{9-11}$. Solid state replacement has also been reported for GaAs nanowires grown with a $\mathrm{Au}$ catalyst ${ }^{12}$, but this case differs significantly from the comparatively large volume of metal available in a deposited contact, as will be shown below. For some of the systems, a distinct epitaxial relationship between the phases was reported, while the absence of an epitaxial relationship was only reported for one specific case ${ }^{7}$. 
For the GaAs-Au based systems, a detailed structural analysis of the newly formed metal phase nanowire and the reaction dynamics during its formation is still lacking. It is also of interest whether there is an induced crystal orientation relationship between the two phases. An earlier study on GaAs-Au with a Ni/Ge/Au metal stack reported diffusion of Ga into the Au and abrupt transitions based on scanning electron microscopy (SEM) observations ${ }^{9}$. Additionally, studies on thin-film based GaAs-Au systems have shown that: i) As evaporates into the vacuum/atmosphere ${ }^{13,14}$; ii) $\mathrm{Ga}$ dissolves in the $\mathrm{Au}$ until it reaches a temperature dependent solubility limit, at which point the reaction stops ${ }^{14,15}$; and iii) that the slowest reaction direction is normal to the $\{111\}$ GaAs crystal planes, with a preference for forming As-terminated $\{111\}$ surfaces $^{16,17}$.

Here, we report a detailed in situ study of the crystal structure development of the heat-induced solid state replacement in the GaAs-Au nanowire system using atomic number contrast in highangle annular dark field (HAADF) scanning transmission electron microscopy (STEM). The pure GaAs-Au system (i.e. without any intermediate metal stacks) was chosen as a model system. No fixed orientation relationship between the newly formed, one-dimensional Au phase and the GaAs phase was found. Additionally, by studying the process in situ for different nanowires, and for a broad range of temperatures, the structure of the growth front could be identified down to the atomic level, as well as the reaction kinetics of the replacement process.

The GaAs nanowires used in this study were grown on Si using self-catalyzed vapor-liquidsolid growth in a molecular beam epitaxy system (for details see Supporting Information S1) ${ }^{18,19}$.

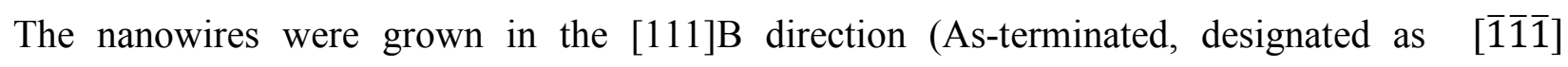
hereafter), and have a hexagonal cross-section with $\{1 \overline{1} 0\}$ surface facets. The in situ TEM specimens were prepared by dispersing the nanowires in isopropyl alcohol, and then micro- 
pipetting the solution onto a heating holder chip (DENS Solutions) with several $50 \mathrm{~nm}$ thick SiN windows (see Figure 1a). Contacts to the nanowires were made by depositing Au by sputtercoating and using electron beam lithography (EBL) to define the contact patterns. For nanowires on off-center windows (NW-A and NW-B below), the temperatures have been scaled by a calibration factor as supplied by the manufacturer. These calibration factors have an uncertainty of about $10 \%$, but the high measurement precision of the relative temperatures is retained, which is the most important metric for the reaction kinetics analysis performed in this Letter. Due to the design of the chip, temperature changes were near instantaneous (up to $200^{\circ} \mathrm{C} / \mathrm{ms}$ ) compared to the STEM image acquisition time.

(a)

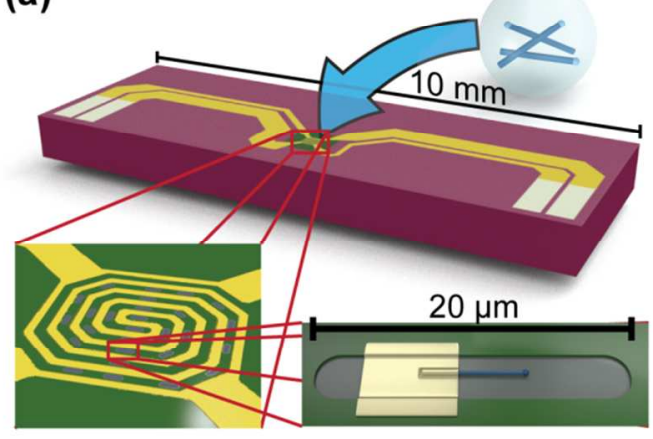

(b)

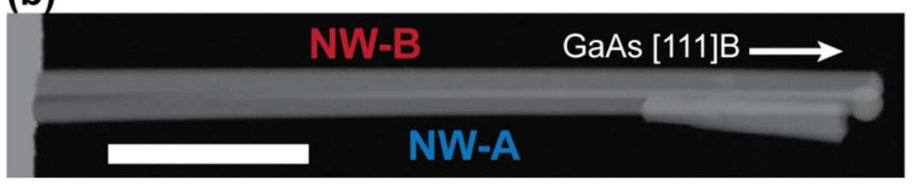

(c)

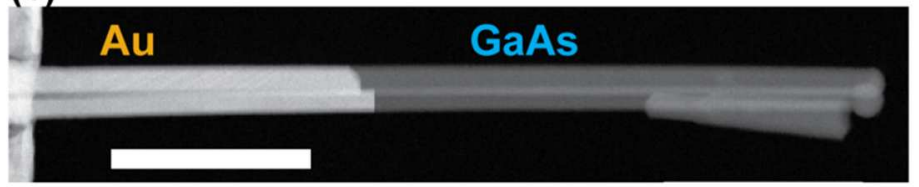

Figure 1: (a) Schematic of the specimen preparation for in situ STEM heating. The GaAs nanowires are dispersed on the heating chip, before Au contacts are fabricated by EBL over part of a nanowire lying on a SiN window. (b, c) HAADF STEM images showing two nearly parallel nanowires (NW-A and NW-B) (b) before and (c) after partial heat-induced replacement by Au. The scale bars are $500 \mathrm{~nm}$.

The nanowires were studied using a double-corrected JEOL ARM 200F TEM/STEM. Figure $1 \mathrm{~b}$ and 1c show HAADF STEM images of a specimen with two nearly parallel nanowires 
(referred to as NW-A and NW-B), before and after in situ heat treatment. The dominant contrast in the HAADF imaging mode is due to the atomic number difference, so the GaAs and Au phases can easily be distinguished. A video montage of the heat treatment is available in Supporting Information video S1. Inspection before the heat treatment showed that the nanowires were zinc blende GaAs with some twinning planes and short wurtzite segments near the nanowire tip, as is common for such nanowires ${ }^{20}$. The heat treatment was halted before this defected region was reached. Intensity line-profiles across the HAADF STEM images of the nanowires (not shown) reveal that the $\mathrm{Au}$ phase kept the hexagonal cross-section of the original nanowire, consistent with observations performed by SEM (not shown).

Crystal orientation mapping of the nanowires was performed after the heat treatment using a JEOL 2100F TEM equipped with a scanning precession electron diffraction system (Nanomegas ASTAR $)^{21}$. The analysis of the crystal orientation mapping data was done by first indexing the orientation of the dominant phase for each scan pixel using the Nanomegas software suite, and then inspecting the resulting orientation maps using the software package MTEX ${ }^{22}$. The orientation measurements were performed at $240{ }^{\circ} \mathrm{C}$, and the results reveal that there is no clear, fixed relationship between the crystallographic orientation of the Au and GaAs phases (Figure 2). Selected-area electron diffraction analysis at room temperature of other heat-treated Au-GaAs nanowires gave similar results (not shown). It is assumed that the orientation of whichever grain from the polycrystalline deposition that becomes dominant in the initial stage of the replacement determines the prevailing orientation. A deposition method that controls the initial crystal orientation of the $\mathrm{Au}$ on the nanowire is required to achieve full control of the Au-GaAs interface formation. In Figure $2 b-e$, the crystal orientations parallel to specific directions have been colorcoded according to the color key in Figure $2 \mathrm{~g}$. These directions were chosen to be normal to the original GaAs nanowire surface facets and parallel to its growth direction, as indicated in 
Figure 2f. These maps show that the surface facets of the Au phase do not in general correspond to any low-order crystal planes. The orientation mapping also reveals the presence of $\{111\}-$ twins in the Au phase in both nanowires, as exemplified by the pole figure in Figure $2 \mathrm{~h}$ of the two dominant $\mathrm{Au}$ orientations in NW-B. Due to the relative orientation of the twinning planes to the electron beam in NW-B, the twins in this nanowire are clearly visible in the HAADF STEM images (indicated by red triangles in Figure 2a). The formation of these twins could therefore be observed in situ during the heat treatment, and they were found to always nucleate at or near the vacuum-Au-GaAs triple-point. They are therefore growth twins and not deformation twins as more commonly observed for $\mathrm{Au}$ nanowires ${ }^{23}$. Once formed, the twins stay fixed during subsequent heating and cooling. From these observations, it is clear that the Au phase of the nanowire remained solid for the duration of the experiment. The Au phase twinning density in NW-B decreased as the temperature was increased, which suggests a temperature dependence of the twin formation. The twinning of the Au phase was found to affect the replacement rate in NW-B, which at times gave rise to a staggered growth front across the twin boundaries (see Supporting Information video S1). The effect of relative orientation on the replacement rate will be discussed further below. Energy dispersive x-ray spectroscopy (EDX) of reacted nanowires showed Ga concentrations below the detection limit ( 1 at.\%), indicating that the Ga diffusion in the $\mathrm{Au}$ is fast compared to the replacement rate. 

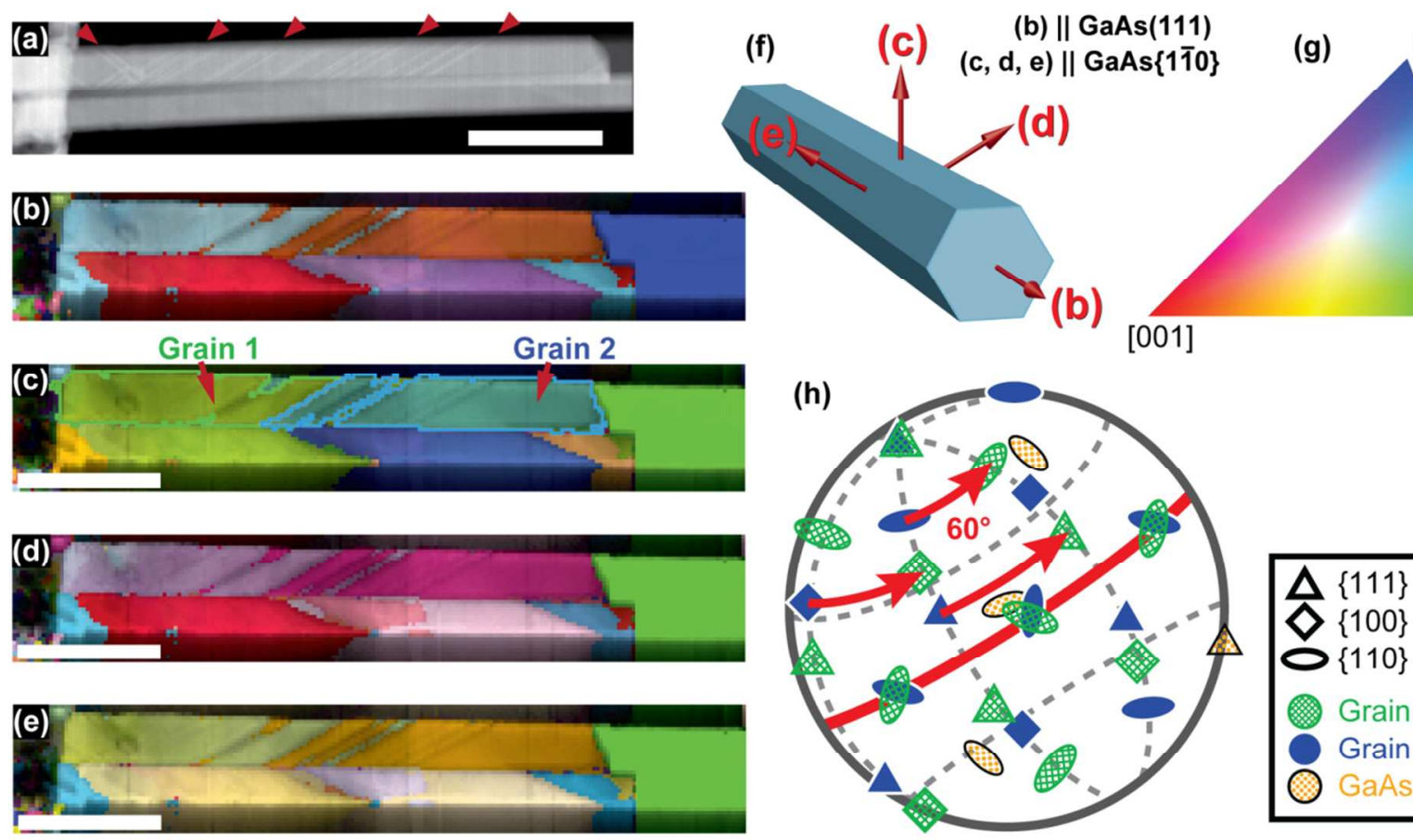

$\Delta\{100\}$

$\circlearrowright\{110\}$

Figure 2: (a) HAADF STEM close-up of the Au phase of NW-A and NW-B where twins in NW$\mathrm{B}$ are directly visible. Red triangles mark the location and direction of some of the twins. (b-e) Crystal orientation maps taken from an angle similar to that in (a). Each map shows the indexed crystal orientations parallel to the directions indicated in (f). The crystal orientation is colorcoded according to the color key shown in (g). (h) Pole figure of GaAs and grains 1 and 2 as indicated in (c). The scale bars in (a-e) are $200 \mathrm{~nm}$.

The stability of the specimen holder and the microscope allowed for lattice-resolved high resolution STEM (HRSTEM) during the replacement process (Figure 3a-e). The Au-GaAs interface mainly followed the GaAs (111) plane (i.e. normal to the nanowire growth direction), but other interface orientations were also observed for shorter periods of time. This is in agreement with thin-film studies, which show that the slowest direction for Au-induced decomposition of GaAs is normal to the $\{111\}$-planes ${ }^{16,17}$. The (111) interface is also the interface-plane with the smallest cross-sectional area for these nanowires. In all cases, the 
interface was abrupt, with little or no observable in-diffusion of Au into the GaAs ahead of the Au-GaAs interface. The in situ HRSTEM observation revealed that the replacement happens one GaAs bi-layer at a time (see Supporting Information video S2, and Figure 3c-e), which differs from the ledge migration process observed for the solid state replacement of $\mathrm{ZnO}$-In nanowires ${ }^{7}$. For the majority of the observations, the interface seems to consist of a complete bi-layer. However, it is not possible to discount partial dissolution of the Ga-columns, which should be considered likely due to the prevalence of the $(2 \times 2)$ vacancy surface reconstruction of the $\operatorname{GaAs}(111) \mathrm{A}$ interface to vacuum/air ${ }^{24}$.
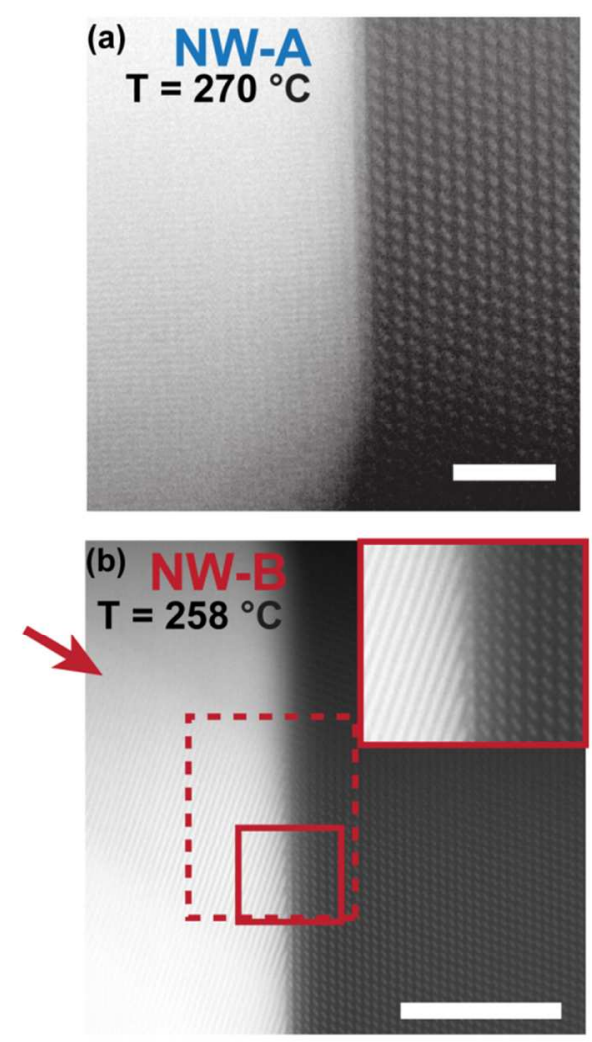
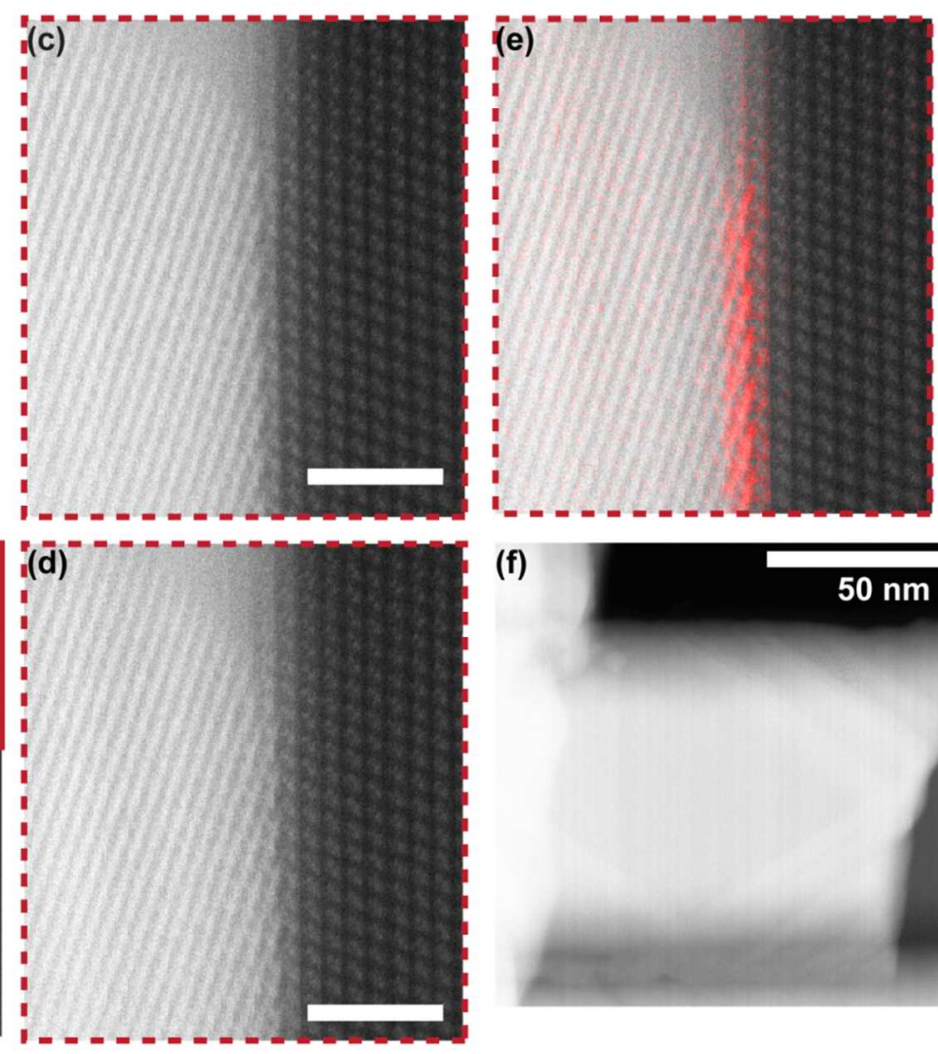

(f)

$50 \mathrm{~nm}$

Figure 3: (a, b) HAADF HRSTEM images showing the interface for (a) NW-A and (b) NW-B at elevated temperatures. (a) is a single frame and (b) is the sum of seven frames. The specimen was tilted such that the GaAs phase was on a [1ํㅣㄹ zone-axis. The red arrow in (b) marks a twin 
boundary, which can be observed since one of the grains is partially aligned along one crystal axis. The inset in (b) is a magnified view of the region highlighted by the solid, red rectangle. (c, d) Two consecutive frames from the time series in Supporting Information video S2, showing the electron beam induced bi-layer replacement. The approximate area is outlined by the dashed, red rectangle in (b). To highlight the change between the frames in (c, d), (e) shows again the frame in (d), with the intensity difference between the frames (c, d) highlighted in red. Scale bars in (ad) are $2 \mathrm{~nm}$. (f) HAADF STEM overview image showing the locally progressed interface due to electron beam induced replacement during HRSTEM imaging (c-e).

At approximately $260^{\circ} \mathrm{C}$, an in situ observable rate of replacement was achieved $(\sim 2 \AA / \mathrm{min}$, trackable within a field-of-view of $160 \times 160 \mathrm{~nm}^{2}$ ). If the electron beam was scanned over a limited interface area at this temperature, a higher replacement rate was observed (Figure 3c-f, Supporting Information video S2). The increase in reaction rate induced by the electron beam is apparent from the clear difference in interface progression for the exposed and unexposed regions (Figure 3f). The probe current was measured to $30 \mathrm{pA}$, giving a frame-averaged current density of roughly $0.5 \mathrm{pA} / \mathrm{nm}^{2}$ during high-resolution imaging. Interestingly, this increased replacement rate was limited to the regions near the area exposed to the electron beam, and did not extend across the entire nanowire cross-section. This indicates that each bi-layer replacement is not strictly limited by a nucleation step, because any nucleation event in the exposed region would then lead to the replacement of the entire GaAs bi-layer. Upon continued heating, the continuous (111) interface eventually recovered across the entire nanowire diameter.

To quantitatively study the temperature dependence of the replacement reaction, 33 in situ HAADF STEM time series were recorded, each at a different fixed temperature. The first time series was recorded at $264^{\circ} \mathrm{C}$, and the temperature was then increased by $3{ }^{\circ} \mathrm{C}$ for each step, up 
to $354{ }^{\circ} \mathrm{C}$ for the last time series (see temperature profile in Figure $4 \mathrm{a}$ ). To facilitate the wide range of observed interface velocities (3 - $300 \AA / \mathrm{min})$, three different STEM magnifications were used $(200 \mathrm{kX}, 400 \mathrm{kX}$, and $800 \mathrm{kX})$. The magnification changes were performed at 300 and $312{ }^{\circ} \mathrm{C}$, as indicated in Figure 4a. A time series was recorded before and after each magnification change at the same temperature, in order to correct for any calibration errors. The observed replacement rates are consistent with the lower limit of those reported in an earlier study, where velocities in the range of $300-9000 \AA /$ min were reported at $360{ }^{\circ} \mathrm{C}^{9}$.
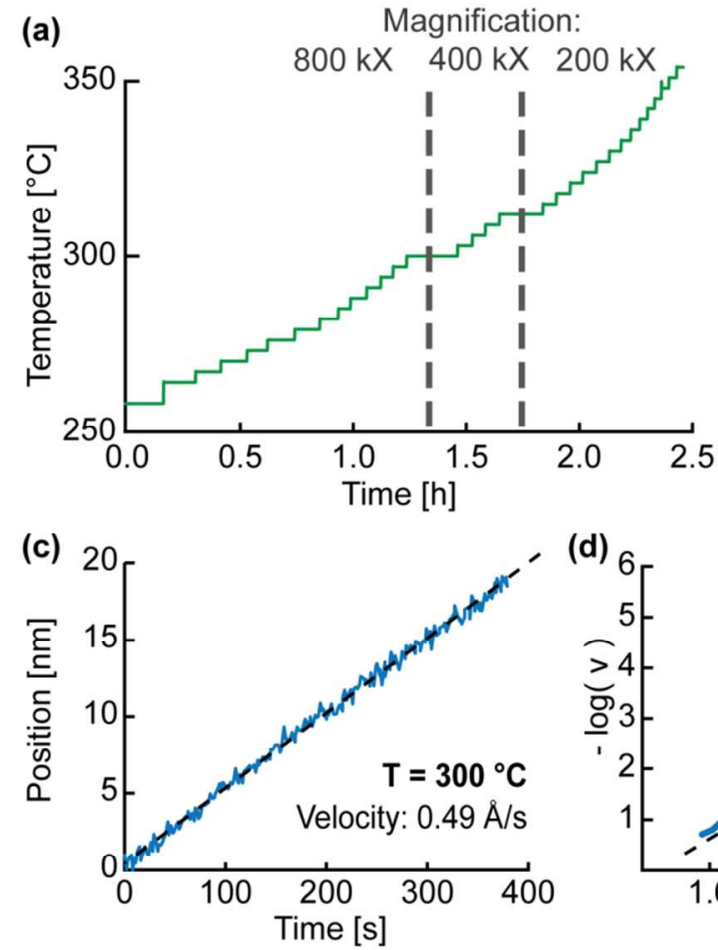

(d)
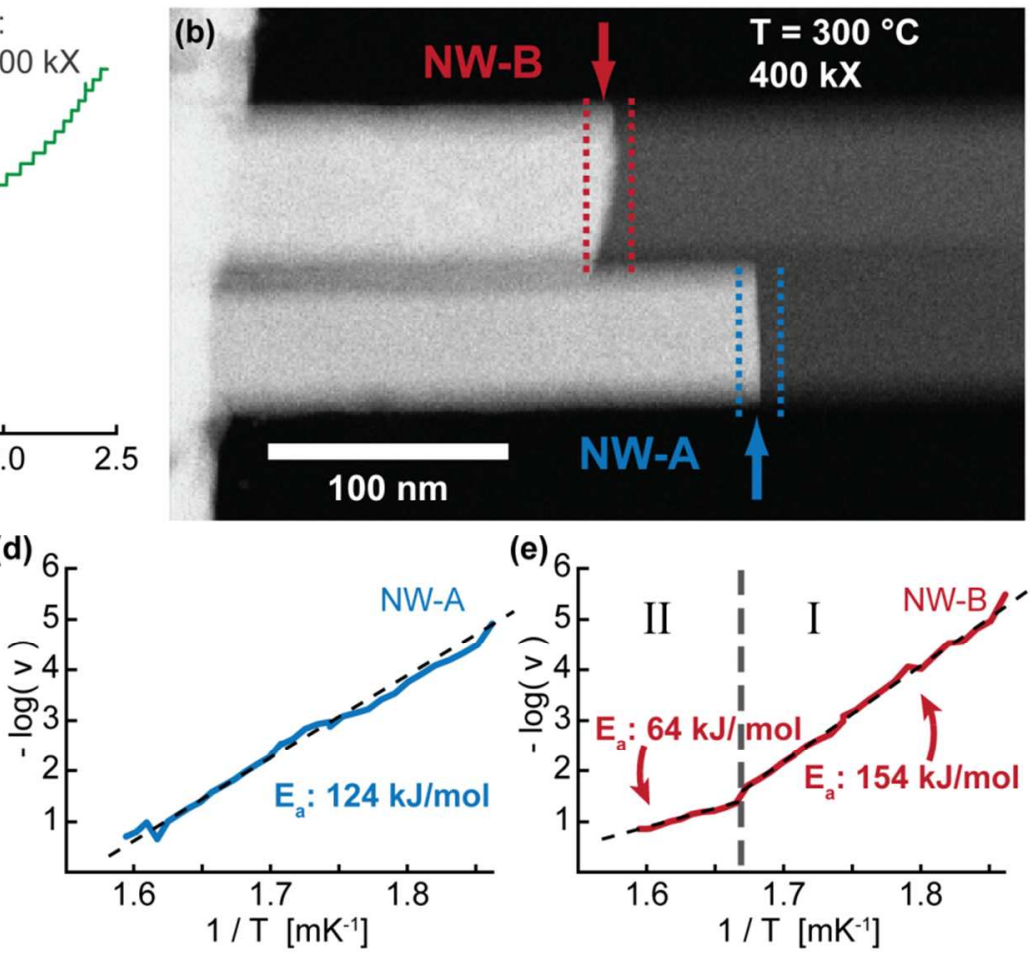

Figure 4: (a) Measured temperature profile during the kinetics measurement. The two magnification changes are indicated by vertical dashed lines. (b) HAADF STEM image illustrating the interface tracking process. The dashed lines indicate the interfaces' positions at the start and end of the time series, and the arrows indicate the positions of the interfaces as found by the tracking algorithm. (c) Position vs. time plot for NW-A in the time series shown in (b). 
The regression line and extracted interface velocity are indicated. (d, e) Arrhenius plots for NWA and NW-B, respectively. Regression lines (dashed) and extracted activation energies are indicated. In (e), two different regimes are labeled, each with a separate regression line and extracted activation energy.

For each time step, the position of the interface was tracked with sub-pixel accuracy using a center of mass algorithm, implemented in the HyperSpy analysis toolset ${ }^{25}$ (Figure $4 \mathrm{~b}$, see Supporting Information S2 for details). The time dependence of the position was used to extract an interface velocity for each temperature by least-squares linear regression (Figure 4c). The complete fitting results and time series statistics are given in Supporting Information Tables S1 and S2. The standard deviation of the regression was typically two orders of magnitude smaller than the fitted value. The linearity of the data was also assessed by fitting a second order polynomial, which were then evaluated on its reduction of fitting error compared to the first-order case (for details see Supporting Information Table S3). In general, it was found that the second order polynomial was able to add very little fitting accuracy, except in one case: NW-B's replacement experienced a significant acceleration during the time series at $327^{\circ} \mathrm{C}$. This acceleration coincides with the change of the dominant twin in NW-B, i.e. the change from grain 1 to grain 2 in Figure 2c (see also Supporting Information video S1).

Finally, the temperature dependence of the interface velocity was analyzed by the logarithmic Arrhenius equation:

$$
\ln v=\ln A-\frac{E_{a}}{k_{B}}\left(\frac{1}{T}\right)
$$

where $v$ is the interface velocity, $E_{a}$ is the activation energy, $\mathrm{T}$ is the absolute temperature, $k_{B}$ is Boltzmann's constant, and A is a prefactor (often called the frequency factor). The equation was fitted using least squares linear regression to extract the effective activation energy of the 
nanowire replacement reaction. For NW-A (Figure 4d) the activation energy was found to be $124 \mathrm{~kJ} / \mathrm{mol}$. For NW-B (Figure 4e), two distinct regimes could be identified. In regime I, the replacement reaction had slightly higher activation energy than that of NW-A $(154 \mathrm{~kJ} / \mathrm{mol})$. Regime II corresponds to the time after the acceleration of the reaction at $327^{\circ} \mathrm{C}$ as discussed above, i.e. approximately when grain 2 in Figure 2c becomes the dominant twin. After this initial velocity increase, a lower activation energy was found $(64 \mathrm{~kJ} / \mathrm{mol})$. This indicates that both the reaction rate and the activation energy depend on the relative orientation of the $\mathrm{Au}$ and GaAs phases. Together with the interface polarity effect seen in thin-films ${ }^{15,16}$, this could explain the large variation in the reaction rates found by Orrù et al. ${ }^{9}$. The estimated activation energies are lower than those found for the $\mathrm{ZnO}-\mathrm{In}$ nanowire system $(325.2 \text { and } 188.7 \mathrm{~kJ} / \mathrm{mol})^{7}$. The accuracy of the activation energies as found by the Arrhenius plot is robust against errors in both the spatial calibration (no effect) and temperature calibration $(<2 \%$ effect for the data presented here) as long as the relative differences are precisely determined. A detailed derivation of the accuracy is included in the Supporting Information S3.

NW-A and NW-B were connected to a large Au reservoir during the entire heat treatment. This reservoir acts both as a constant source of $\mathrm{Au}$, and a sink for $\mathrm{Ga}$ to diffuse into. When this connection is broken as it did for a different nanowire (NW-C, see Figure 5a and Supporting Information video S3), the dynamics of the exchange process differ distinctly. The cause of the disconnection of NW-C could not be identified as it occurred while the interface was underneath the thick Au layer, and therefore not observable in the TEM. Despite this disconnection, the replacement process continued with the limited amount of Au available. EDX analysis of the metal phase shows that the metal segment is comprised of $\mathrm{Au}$ and $\mathrm{Ga}$ (inset in Figure 5c), reinforcing the earlier expectations that $\mathrm{As}$ evaporates ${ }^{13,14}$, and that the $\mathrm{Ga}$ dissolves into the $\mathrm{Au}^{9}$, 14, 15. For this nanowire, the metal phase became liquid over time, as the temperature, and 
consequently Ga content, was increased. Quantitative EDX analysis indicates an average Ga concentration of 50 at. $\%$ in the metal phase depicted in Figure 5c. The volume of the disconnected metal segment increased as the replacement processes progressed, as would be expected due to the influx of Ga. This enrichment of Ga causes a reduction of the reaction rate as it approaches the solubility limit at a given temperature ${ }^{14}$. This was observed as a marked decrease in replacement rate over time, almost until the point of a complete halt. A further increase in the temperature caused the replacement reaction to continue, with again a decreasing reaction rate over time. This observation is relevant for GaAs nanowires grown with $\mathrm{Au}$ as a growth catalyst, as they should only undergo a limited amount of solid state replacement if they are reheated, due to the limited ability of the growth catalyst to dissolve Ga. It is also relevant for nanowire device designs, where greater fabrication control of the Au-GaAs junction can be achieved by controlling the volume of deposited metal compared to that of the nanowires prior to annealing. This will in essence make the reaction self-limited. Upon cooling, the metal phase in NW-C mainly recrystallized into the AuGa intermetallic phase ${ }^{26}$, as determined by electron diffraction, corresponding well with the Ga content found by EDX. 
(a)
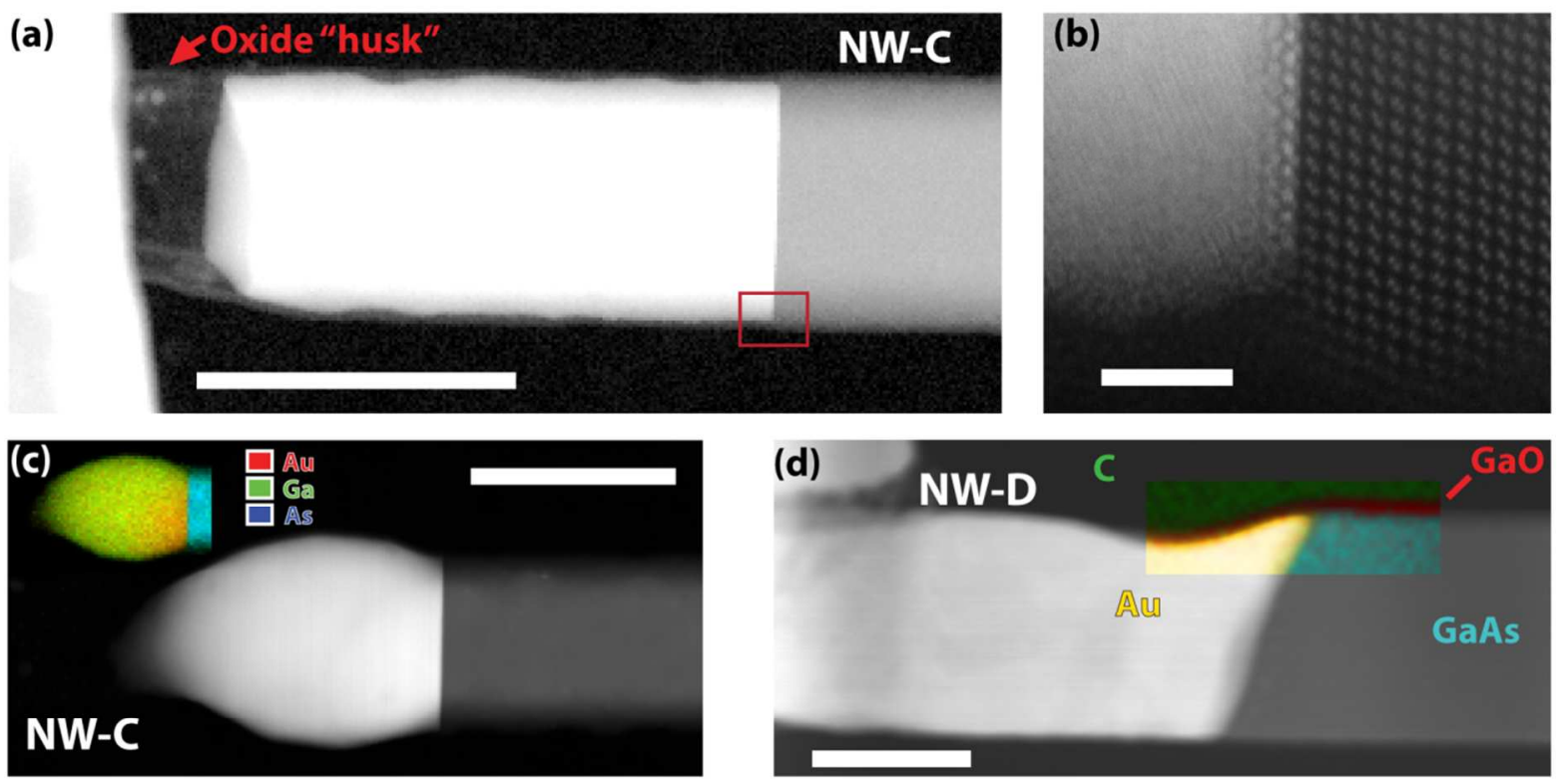

GaO

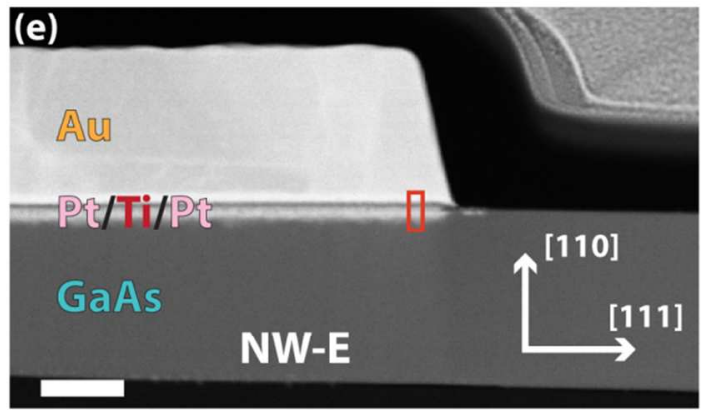

(f)

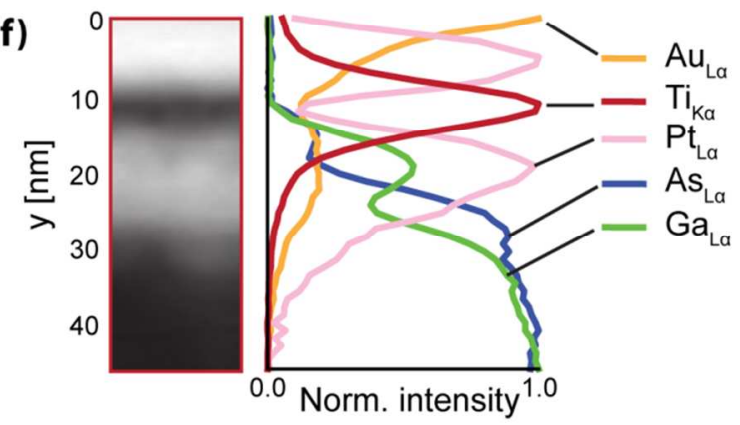

Figure 5: HAADF STEM images of: (a) NW-C after the metal segment appeared from underneath the Au-deposit. The contrast response of the image has been adjusted to better show the encasing $\mathrm{GaO}_{\mathrm{x}}$. (b) HRSTEM of the interface as indicated by the red box in (a). (a) and (b) were acquired at room temperature. (c) NW-C after the breakdown of the encasing oxide (image taken after solidification by cooling to $120^{\circ} \mathrm{C}$ ). Inset: EDX element map. (d) NW-D in a crosssection extracted by focused ion beam (FIB), with an overlaid, color-coded EDX phase map. The phases were mapped by blind source separation ${ }^{27}$, and show the presence of $\mathrm{GaO}_{\mathrm{x}}$ also covering the Au segment. (e) NW-E in a FIB cross-section. (f) Integrated EDX line profiles of the area outlined by the red box shown in (e). Scale bars are $100 \mathrm{~nm}$ except in (b) where it is $2 \mathrm{~nm}$. 
The Au-GaAs interface for NW-C differed distinctly compared to those of NW-A and NW-B. By HRSTEM (Figure 5b) it can be seen that the As atomic columns of the GaAs bilayer at the interface have a higher intensity. This is different from what was observed for NW-A and NW-B, which is likely due to the fact that NW-C has its (111)B interface towards the Au instead of a (111)A interface. This shows that Au substitutes the top As layer either partially or completely on the (111)B interface. While Figure 5b was taken at room temperature, identical features are also visible when the metal segment is in the liquid phase. The As-layer replacement in NW-C is in agreement with density functional simulations of Au-GaAs(111)B interfaces under Ga-rich conditions $^{28}$.

As shown above, NW-A and NW-B kept their size and shape during the solid state replacement. Additionally, the crystal orientation mapping in Figure 2b-e revealed that the surface facets of the Au phase do not correspond to any low-order crystal planes. As Au is highly mobile at the elevated temperatures used, it would be natural to expect that lower energy surface facets would form (typically $\{111\}$ facets $^{29}$ ). EDX spectral imaging reveals that the original encasing $\mathrm{GaO}_{\mathrm{x}}$ layer remains after $\mathrm{Au}$ replacement of the GaAs nanowire (Figure 5a, d). We therefore suspect that the gallium oxide enforces its shape on the Au, either by sterically hindering it from expanding, or by reducing the interfacial energy such that the Au is prevented from shrinking. This is corroborated by the fact that the newly formed Au phase follows the existing roughness of the $\mathrm{GaAs} / \mathrm{GaO}_{\mathrm{x}}$ when replacing the GaAs (see Supporting information video S1 and S3). It should be pointed out that for NW-C, the liquid metal phase was able to expand beyond the boundaries of the original GaAs nanowire (Figure 5c) because the $\mathrm{GaO}_{\mathrm{x}}$ layer broke up and coalesced at these elevated temperatures (see Supporting information video S3).

To demonstrate whether the above findings could also take place when using common metallic intermediate stacks and conventional process equipment, results from additional nanowires 
(NW-D and NW-E) are shown in Figure 5d-f. These nanowires were contacted by a Pt/Ti/Pt/Au metal stack $(5 / 10 / 5 / 150 \mathrm{~nm})$ and annealed in a rapid thermal annealing oven under vacuum at $400{ }^{\circ} \mathrm{C}$ for $30 \mathrm{~s}$, similar to what was done in other studies ${ }^{30,31}$. For such a stack configuration and annealing regime, $\mathrm{Au}$ is not expected to enter the nanowire in any significant amounts, mainly due to Ti acting as a diffusion barrier ${ }^{32}$. However, for several nanowires, Au-GaAs replacement is still observed by SEM, as confirmed by cross-sectional STEM of NW-D (Figure 5d). This is likely caused by either a broken barrier or by Au diffusing around the barrier. Such nanowires show similar features as the Au-GaAs model case, albeit with necking (reduction in diameter). The necking is attributed to a limited diffusion of $\mathrm{Au}$ into the nanowire, rather than limited diffusion of Ga across the barrier. This is supported by the fact that EDX shows little or no traces of $\mathrm{Ga}$ in the metal nanowire phase. Samples where the barrier behaved as expected were also observed (NW-E, Figure 5e-f). In such cases, a layer of mixed polycrystalline intermetallics formed along the interface.

In conclusion, we have reported on the in situ tracking of the thermally induced replacement of GaAs nanowires by $\mathrm{Au}$, down to atomic resolution. This allowed us to determine that the reaction occurs one GaAs bi-layer at a time. Two separate regimes have been identified: a virtually endless $\mathrm{Au}$ reservoir/Ga sink, and a limited $\mathrm{Au}$ volume. For the endless reservoir configuration, the in situ tracking allowed for the quantification of the reaction rate, as well as its temperature dependence. Reaction rates in the range of 3-300 $\AA / \mathrm{min}$ were observed in the temperature range 264-354 ${ }^{\circ} \mathrm{C}$. The metal phase remained solid as long as it was connected to the Au reservoir. Lattice-resolved imaging and crystal orientation mapping ruled out the existence of a fixed orientation relationship between the $\mathrm{Au}$ and GaAs parts of the nanowires. The relative orientation of the GaAs and Au phases seems to influence both the reaction rate, and its temperature dependence. Effective activation energies of the replacement reaction were found to be 
$124 \mathrm{~kJ} / \mathrm{mol}$ for one nanowire and 64 and $154 \mathrm{~kJ} / \mathrm{mol}$ for two distinct regimes in another nanowire. The Au phases contained a varying density of $\{111\}$ growth twins, which remained fixed for the entirety of the replacement process. The nanowires retained their hexagonal cross-section during and after the replacement. When the Au reservoir was limited, a decrease in the reaction rate was observed as the Ga content in the Au reservoir increased. In this case, the metal phase can also become liquid. With this improved insight into the solid exchange process, it should be possible to further optimize the controlled formation of reproducible, high-quality Au-GaAs junctions within nanowires.

\title{
ASSOCIATED CONTENT
}

Supporting Information. Detailed description of nanowire synthesis; a description of the interface tracking algorithm; detailed derivation of the accuracy of found activation energies; detailed statistics of time series and their regression results; and videos showing the in situ replacement in NW-A, NW-B and NW-C. The Supporting Information is available free of charge on the ACS Publications website at DOI: 10.1021/acs.nanolett.0X00000.

\section{AUTHOR INFORMATION}

\section{Corresponding Author}

*E-mail: a.helvoort@ntnu.no

\author{
Author Contributions \\ VTF conceived the idea of the study together with JH. VTF and ATJvH planned and developed \\ the study. The nanowires were grown and characterized with SEM by AMM and DLD. Specimen
}


preparation was done by VTF and JH. Preliminary in situ TEM results were obtained by VTF and GD. VTF performed all further TEM, SEM, FIB and the data analysis. VTF drafted the manuscript together with ATJvH. All co-authors gave feedback during the writing process.

\section{Notes}

The authors declare no competing financial interest.

\section{ACKNOWLEDGMENT}

The Research Council of Norway is acknowledged for the support to the Norwegian Microand Nano-Fabrication Facility, NorFab (197411/V30), the FRINATEK program (214235) and the NORTEM project (197405). G.D. and C.D. acknowledge funding from ERC under grant number 259619 PHOTO EM. C.D. acknowledges financial support from the EU under grant number 312483 ESTEEM2.

\section{REFERENCES}

1. Yan, R.; Gargas, D.; Yang, P. Nature Photonics 2009, 3, (10), 569-576.

2. Joyce, H. J.; Gao, Q.; Hoe Tan, H.; Jagadish, C.; Kim, Y.; Zou, J.; Smith, L. M.; Jackson, H. E.; Yarrison-Rice, J. M.; Parkinson, P.; Johnston, M. B. Progress in Quantum Electronics 2011, 35, (2-3), 23-75.

3. $\quad$ Yang, P.; Yan, R.; Fardy, M. Nano Letters 2010, 10, (5), 1529-1536.

4. $\quad$ Wu, Y.; Xiang, J.; Yang, C.; Lu, W.; Lieber, C. M. Nature 2004, 430, 61.

5. Lin, Y.-C.; Lu, K.-C.; Wu, W.-W.; Bai, J.; Chen, L. J.; Tu, K. N.; Huang, Y. Nano Letters 2008, 8, (3), 913-918.

6. Burchhart, T.; Lugstein, A.; Hyun, Y. J.; Hochleitner, G.; Bertagnolli, E. Nano Letters 2009, 9, (11), 3739-42.

7. $\quad$ Wang, S.-C.; Lu, M.-Y.; Manekkathodi, A.; Liu, P.-H.; Lin, H.-C.; Li, W.-S.; Hou, T.-C.; Gwo, S.; Chen, L.-J. Nano Letters 2014, 14, (6), 3241-3246.

8. Kral, S.; Zeiner, C.; Stöger-Pollach, M.; Bertagnolli, E.; den Hertog, M. I.; Lopez-Haro, M.; Robin, E.; El Hajraoui, K.; Lugstein, A. Nano Letters 2015, 15, (7), 4783-4787.

9. Orrù, M.; Rubini, S.; Roddaro, S. Semiconductor Science and Technology 2014, 29, (5), 054001. 
10. Orrù, M.; Piazza, V.; Rubini, S.; Roddaro, S. Physical Review Applied 2015, 4, (4), 044010.

11. Gutsche, C.; Lysov, A.; Regolin, I.; Brodt, A.; Liborius, L.; Frohleiks, J.; Prost, W.; Tegude, F. J. Journal of Applied Physics 2011, 110, (1), 014305.

12. Persson, A. I.; Larsson, M. W.; Stenstrom, S.; Ohlsson, B. J.; Samuelson, L.; Wallenberg, L. R. Nat Mater 2004, 3, (10), 677-681.

13. Sebestyen, T.; Menyhard, M.; Szigethy, D. Electronics Letters 1976, 12, (4), 96-97.

14. Kinsbron, E.; Gallagher, P. K.; English, A. T. Solid-State Electronics 1979, 22, (5), $517-$ 524.

15. Sebestyen, T.; Mojzes, I.; Szigethy, D. Electronics Letters 1980, 16, (13), 504-505.

16. Bauer, C. L. Surface Science 1986, 168, (1-3), 395-403.

17. Holloway, P. H.; Mueller, C. H. Thin Solid Films 1992, 221, (1-2), 254-261.

18. Munshi, A. M.; Dheeraj, D. L.; Fauske, V. T.; Kim, D. C.; Huh, J.; Reinertsen, J. F.; Ahtapodov, L.; Lee, K. D.; Heidari, B.; van Helvoort, A. T. J.; Fimland, B. O.; Weman, H. Nano Letters 2014, 14, (2), 960-966.

19. Morral, A. F. Selected Topics in Quantum Electronics, IEEE Journal of 2011, 17, (4), 819-828.

20. Krogstrup, P.; Popovitz-Biro, R.; Johnson, E.; Madsen, M. H.; Nygård, J.; Shtrikman, H. Nano Letters 2010, 10, (11), 4475-4482.

21. Moeck, P.; Rouvimov, S.; Rauch, E. F.; Véron, M.; Kirmse, H.; Häusler, I.; Neumann, W.; Bultreys, D.; Maniette, Y.; Nicolopoulos, S. Crystal Research and Technology 2011, 46, (6), 589-606.

22. Bachmann, F.; Hielscher, R.; Schaeben, H. Ultramicroscopy 2011, 111, (12), 1720-1733.

23. Lee, S.; Im, J.; Yoo, Y.; Bitzek, E.; Kiener, D.; Richter, G.; Kim, B.; Oh, S. H. Nature Communications 2014, 5.

24. Woolf, D. A.; Westwood, D. I.; Williams, R. H. Applied Physics Letters 1993, 62, (12), 1370-1372.

25. Peña, F. d. 1.; Burdet, P.; Ostasevicius, T.; Sarahan, M.; Nord, M.; Fauske, V. T.; Taillon, J.; Eljarrat, A.; Mazzucco, S.; Donval, G.; Zagonel, L. F.; Walls, M. hyperspy: HyperSpy 0.8.2, 2015.

26. Elliott, R. P.; Shunk, F. A. Bulletin of Alloy Phase Diagrams 1981, 2, (3), 356-358.

27. Rossouw, D.; Burdet, P.; de la Peña, F.; Ducati, C.; Knappett, B. R.; Wheatley, A. E. H.; Midgley, P. A. Nano Letters 2015, 15, (4), 2716-2720.

28. Shu, H.; Chen, X.; Lu, W. Journal of Applied Physics 2010, 108, (1), 013526.

29. Chen, Y.; Milenkovic, S.; Hassel, A. W. Applied Surface Science 2012, 258, (17), 62246231.

30. Dheeraj, D. L.; Munshi, A. M.; Christoffersen, O. M.; Kim, D. C.; Signorello, G.; Riel, H.; van Helvoort, A. T. J.; Weman, H.; Fimland, B. O. Journal of Crystal Growth 2013, 378, 532-536.

31. Huh, J.; Yun, H.; Kim, D.-C.; Munshi, A. M.; Dheeraj, D. L.; Kauko, H.; van Helvoort, A. T. J.; Lee, S.; Fimland, B.-O.; Weman, H. Nano Letters 2015, 15, (6), 3709-3715.

32. Nebauer, E.; Mai, M.; Würfl, J.; Österle, W. Semiconductor Science and Technology 2000, 15, (8), 818 . 
28

30

31

32

33

34

35

37

38

39

40

41

42

44

45

46

47

48

49

51

52

53

54

55

57

58

59

60 


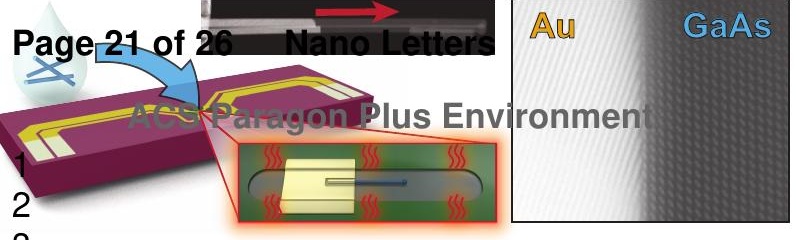


(a) $N W-A$
$T=270^{\circ} \mathrm{C}$

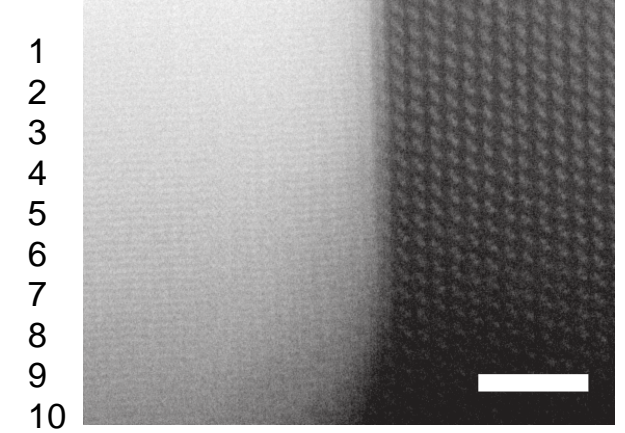

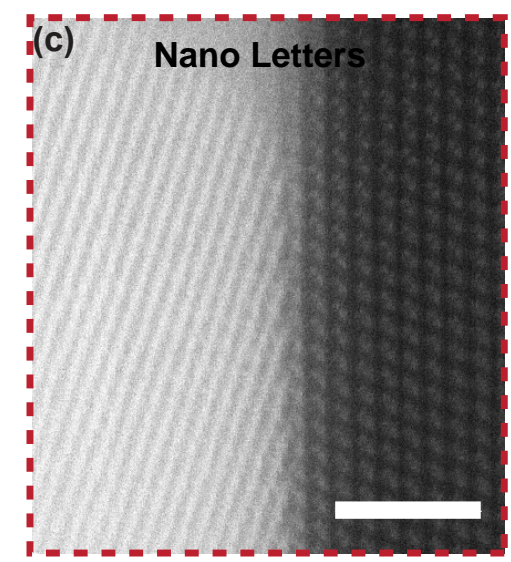

11

12

13

(b) NW-E (d)

$\mathrm{T}=258^{\circ} \mathrm{C}$

15

16

17

18

19

20

21

22

23

24 $\overline{(e)}$

of 26

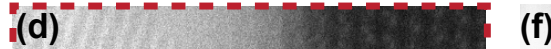

(f)

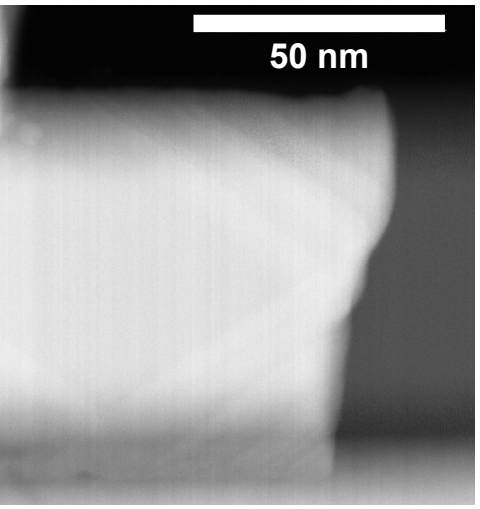




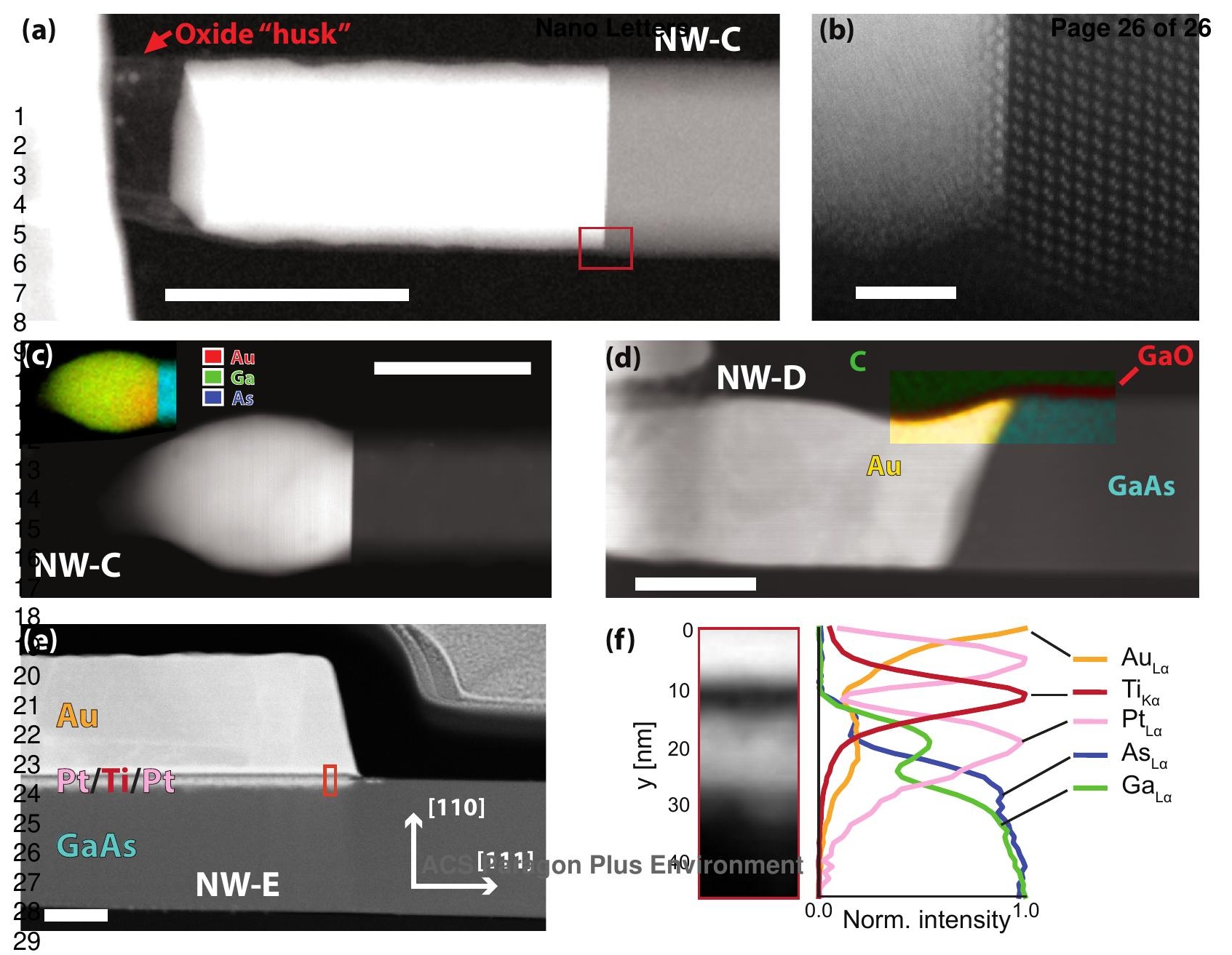

